Collection SFN 9 (2008) 1-17

(C) EDP Sciences, Les Ulis

DOI: $10.1051 / \mathrm{sfn}: 2008002$

\title{
Rappels de cristallographie
}

\author{
P. Bordet \\ Institut Néel, CNRS-UJF, BP. 166, 38042 Grenoble Cedex 9, France \\ e-mail : pierre.bordet@grenoble.cnrs.fr
}

\section{CRISTAUX ET SYMÉTRIE}

\subsection{Introduction}

Lorsqu'on évoque les cristaux, on pense généralement aux spécimens minéralogiques, tels que le quartz ou les gemmes, dont on peut admirer les formes géométriques et les couleurs variées. En fait, la plupart des matériaux solides inorganiques existent naturellement sous forme cristalline. La taille des cristaux est alors de l'ordre du micron, et le matériau se présente sous forme de poudre ou d'agrégats de grains microcristallins, indépendants (dans les poudres), ou liés entre eux (comme dans les métaux).

Ce qui fait la spécificité des cristaux par rapports aux autres états de la matière solide (amorphes, verres), c'est l'ordre à longue distance de l'arrangement atomique. Un cristal peut être considéré comme l'empilement tridimensionnel périodique de "briques" ou mailles élémentaires identiques. Chaque maille est constituée d'un ensemble d'atomes. La description de l'arrangement des atomes au sein d'une seule maille, ainsi que les propriétés géométriques de cette maille et de l'empilement des mailles dans l'espace, fournit une description atomique complète de l'ensemble du cristal. A partir de la connaissance des positions atomiques dans le cristal, on peut envisager de comprendre les propriétés physico-chimiques du matériau.

La nécessité de remplir l'espace à l'aide de briques identiques implique l'existence de propriétés géométriques de symétrie spécifiques de l'état cristallin. Ces propriétés se reflètent notamment dans la forme extérieure des cristaux macroscopiques, caractérisée par l'existence de faces planes faisant entre elles des angles déterminés.

Actuellement, les techniques expérimentales de diffraction des rayons $\mathrm{X}$ et des neutrons, ainsi que la microscopie électronique, ont permis de déterminer avec une étonnante précision l'arrangement structural d'un grand nombre de matériaux cristallisés, allant des minéraux naturels aux céramiques synthétisées en laboratoire, et jusqu'aux molécules biologiques, pour lesquelles on sait maintenant fabriquer des cristaux. La structure en double hélice de l'ADN a ainsi été découverte par des techniques cristallographiques de diffraction des rayons $\mathrm{X}$ sur des cristaux d'ADN.

Dans ce cours, nous ferons un bref rappel des propriétés de symétrie des cristaux, puis nous donnerons une introduction aux méthodes d'analyse cristallographiques aussi bien pour les monocristaux que pour les poudres. Nous nous sommes largement inspirés des documents de cours de F. Boucher et Ph. Deniard (IMN, Nantes) pour sa réalisation.

\subsection{Réseau, éléments de symétrie des cristaux}

\subsubsection{Réseaux}

\section{Réseau Cristallin}

Le réseau cristallin est constitué d'une infinité triplement périodique de points géométriques se déduisant les uns des autres par des translations du type : $u \vec{a}+v \vec{b}+w \vec{c}$, où $\vec{a}, \vec{b}, \vec{c}$ sont trois vecteurs non coplanaires et $\mathrm{u}, \mathrm{v}, \mathrm{w}$ sont des entiers. Chaque point du réseau est appelé nœud.

Maille

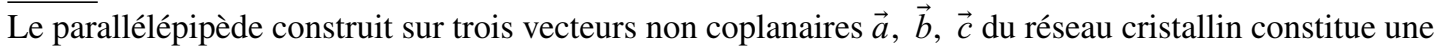
maille du réseau. Si tout nœud du réseau peut être obtenu par combinaison linéaire entière des vecteurs 

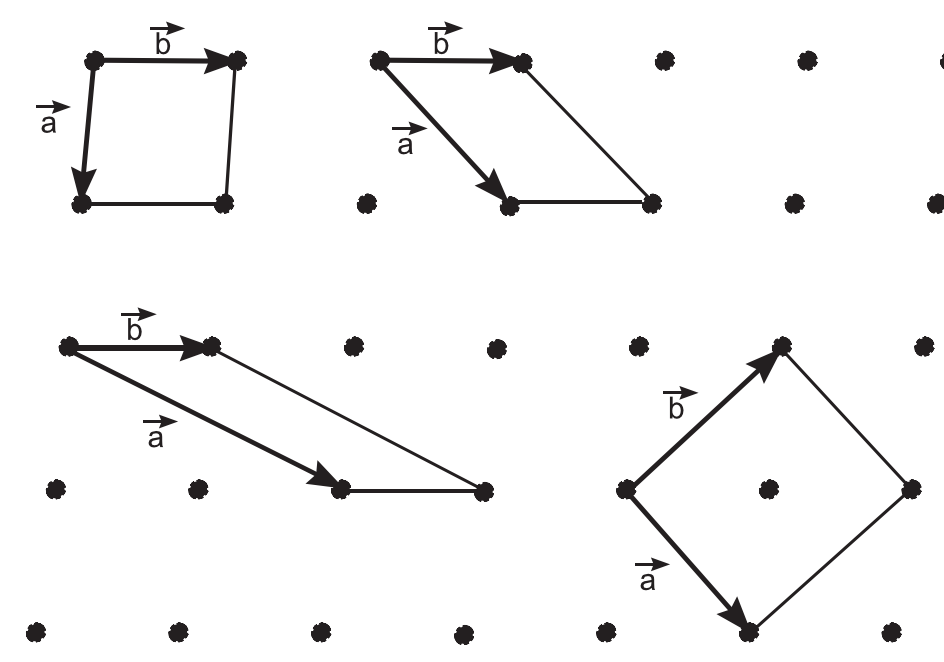

Figure 1. Mailles d'un réseau à 2 dimensions.

de la maille, la maille est dite simple, ou primitive. Sinon, elle est dite multiple. Le volume d'une maille simple contient un seul nœud du réseau. Le volume d'une maille multiple contient un nombre entier de nœuds du réseau. Une maille multiple contenant $n$ nœud du réseau ( $\mathrm{n}$ : entier) est appelée maille multiple d'ordre $\underline{n}$.

Les modules des vecteurs de la maille sont appelés paramètres de maille. Ils sont traditionnellement notés $(\mathrm{a}, \mathrm{b}, \mathrm{c})$. Les angles entres vecteurs de maille sont notés $\alpha, \beta, \gamma$, où $\alpha$ est l'angle entre $\vec{b}$ et $\vec{c}$, etc...

On classifie les cristaux en 7 systèmes cristallins tridimensionnels, caractérisés par la géométrie de leur maille primitive.

$$
\begin{array}{ll}
\text { - triclinique } & \mathrm{a} \neq \mathrm{b} \neq \mathrm{c}, \alpha \neq \beta \neq \gamma \neq 90^{\circ} \\
\text { - monoclinique } & \mathrm{a} \neq \mathrm{b} \neq \mathrm{c}, \alpha=\gamma=90^{\circ} \neq \beta \\
\text { - orthorhombique } & \mathrm{a} \neq \mathrm{b} \neq \mathrm{c}, \alpha=\beta=\gamma=90^{\circ} \\
\text { - quadratique (tetragonal) } & \mathrm{a}=\mathrm{b} \neq \mathrm{c}, \alpha=\beta=\gamma=90^{\circ} \\
\text { - rhomboédrique } & \mathrm{a}=\mathrm{b}=\mathrm{c}, \alpha=\beta=\gamma \neq 90^{\circ} \\
\text { - hexagonal } & \mathrm{a}=\mathrm{b} \neq \mathrm{c}, \alpha=\beta=90^{\circ}, \gamma=120^{\circ} \\
\text { - cubique } & \mathrm{a}=\mathrm{b}=\mathrm{c}, \alpha=\beta=\gamma=90^{\circ} .
\end{array}
$$

Les mêmes définitions s'appliquent au cas à deux dimensions, où le réseau est plan. On présente cidessous un réseau constitué d'un ensemble de points, et quatre mailles de ce réseau. Trois mailles sont primitives, et une est multiple d'ordre 2 (en bas à droite).

On peut vérifier aisément que les surfaces de toutes les mailles primitives sont égales, alors que la surface de la maille d'ordre 2 vaut deux fois celle d'une maille primitive. Chaque maille primitive contient un seul nœud du réseau : elle a en effet 4 nœuds à ses 4 sommets, mais chaque nœud appartient à 4 mailles adjacentes, et doit donc être compté pour 1/4. La maille double contient en plus un nœud en son centre : elle contient donc bien 2 nœuds du réseau.

$\underline{\text { Réseaux de Bravais }}$

A priori, on a un choix infini de mailles de réseaux différentes. Cependant, il est intéressant de reconnaître un certain nombre de réseaux spéciaux, appelés Réseaux de Bravais, qui sont invariants dans des opérations de symétrie. L'utilisation de ces réseaux, permet de mettre en évidence les opérations de symétrie existantes et donc de simplifier la description du cristal. Les réseaux de Bravais sont caractérisés par des rapports particuliers entre les paramètres de maille, et des angles particuliers entre les vecteurs de maille. Ils peuvent correspondre à des mailles multiples. A trois dimensions, on peut définir 14 réseaux de Bravais différents. Ces réseaux se distinguent d'une part par la géométrie de la maille (système cristallin), et d'autre part par le type de centrage, dans le cas des mailles multiples. 


\begin{tabular}{|l|l|l|}
\hline Système & symbole de réseau & géométrie de la maille \\
\hline triclinique & $\mathrm{P}$ & $\mathrm{a} \neq \mathrm{b} \neq \mathrm{c}, \alpha \neq \beta \neq \gamma \neq 90^{\circ}$ \\
\hline monoclinique & $\mathrm{P}, \mathrm{C}$ & $\mathrm{a} \neq \mathrm{b} \neq \mathrm{c}, \alpha=\gamma=90^{\circ} \neq \beta$ \\
\hline orthorhombique & $\mathrm{P}, \mathrm{C}, \mathrm{I}, \mathrm{F}$ & $\mathrm{a} \neq \mathrm{b} \neq \mathrm{c}, \alpha=\beta=\gamma=90^{\circ}$ \\
\hline quadratique & $\mathrm{P}, \mathrm{I}$ & $\mathrm{a}=\mathrm{b} \neq \mathrm{c}, \alpha=\beta=\gamma=90^{\circ}$ \\
\hline rhomboédrique & $\mathrm{P}$ & $\mathrm{a}=\mathrm{b}=\mathrm{c}, \alpha=\beta=\gamma \neq 90^{\circ}$ \\
\hline hexagonal & $\mathrm{P}$ & $\mathrm{a}=\mathrm{b} \neq \mathrm{c}, \alpha=\beta=90^{\circ}, \gamma=120^{\circ}$ \\
\hline cubique & $\mathrm{P}, \mathrm{I}, \mathrm{F}$ & $\mathrm{a}=\mathrm{b}=\mathrm{c}, \alpha=\beta=\gamma=90^{\circ}$ \\
\hline
\end{tabular}

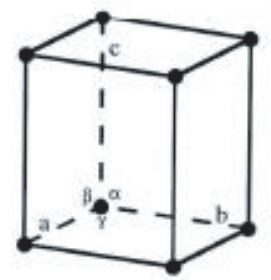

triclinique $\mathrm{P}$

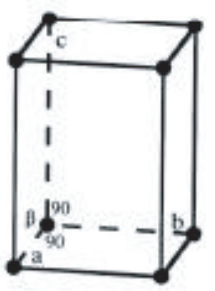

monoclinique $\mathrm{P}$

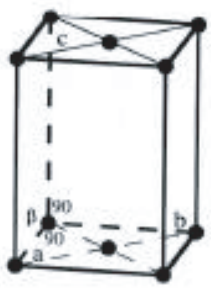

monoclinique $\mathrm{C}$

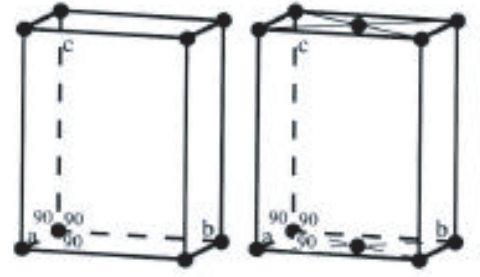

orthorhombique P

orthorhombique C

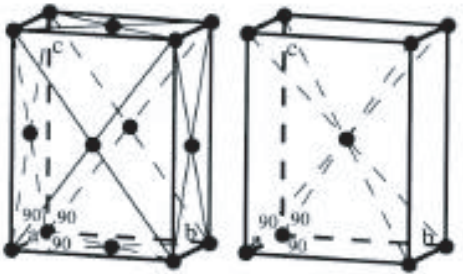

orthorhombique $\mathrm{F}$

orthorhombique I

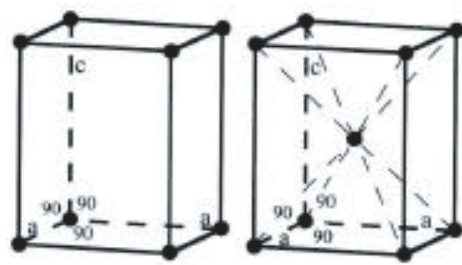

tétragonal $P$

tétragonal I

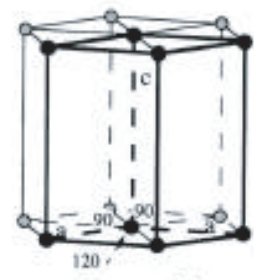

hexagonal P

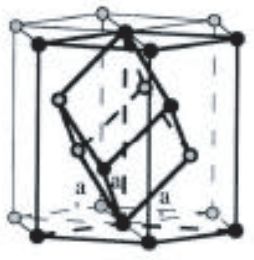

rhombøédrique $\mathrm{R}$

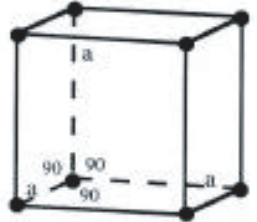

cubique $\mathrm{P}$

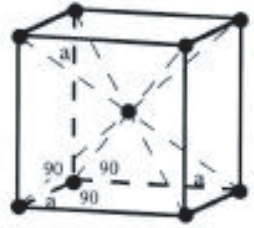

cubique I

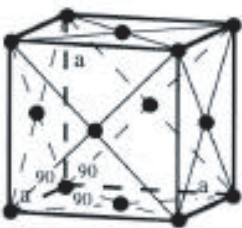

cubique $\mathrm{F}$

Figure 2. Les 14 réseaux de Bravais à 3 dimensions.

On distingue 4 types de centrages différents :

- P : primitif : pas de centrage, la maille est simple, il y a un nœud du réseau par maille.

- I : corps centré : il y a un nœud du réseau au centre de la maille. La maille est double (d'ordre 2), il y a 2 nœuds du réseau par maille. 
- F : faces centrées : il y a un nœud du réseau au centre de chacune des 6 faces de la maille. Chacun de ces 6 nœuds étant partagé entre les faces de deux mailles adjacentes, il y aura en tout $1+3=4$ nœuds du réseau par maille, qui est donc d'ordre 4.

- C : face C centrée. Il y a un nœud du réseau au centre des deux faces perpendiculaires à l'axe c de la maille. Chacun de ces 2 nœuds étant partagé entre les faces de deux mailles adjacentes, il y aura en tout $1+1=2$ nœuds du réseau par maille, qui est donc d'ordre 2 . Notons qu'on définit de même des types de centrages $\mathrm{A}$ ou $\mathrm{B}$, suivant le choix des axes de la maille. Les trois possibilités étant équivalentes, on choisit par convention le centrage $\mathrm{C}$.

Tous les types de centrages ne sont pas applicables à chaque système cristallin. Par exemple, dans un réseau triclinique corps centré, il est toujours possible de trouver une maille primitive plus petite (de volume moitié, en fait) qui contiendra un seul nœud du réseau. L'intérêt de choisir une maille centrée est de mettre en évidence des propriétés de symétrie qui pourraient passer inaperçues en choisissant une maille simple. Les propriétés des 14 réseaux de Bravais en trois dimensions sont résumées sur le tableau et la figure ci-dessous.

\subsubsection{Eléments de symétrie}

On désigne par symétrie l'invariance d'un objet ou d'une structure par rapport à certaines opérations. Une opération de symétrie géométrique est une application de l'espace sur lui-même. Elle transforme un objet en lui-même, sans déformations. On peut représenter une opération de symétrie géométrique par une application affine du type :

$$
\left(\begin{array}{l}
x_{1}^{\prime} \\
x_{2}^{\prime} \\
x_{3}^{\prime}
\end{array}\right)=\left(\begin{array}{lll}
r_{11} & r_{12} & r_{13} \\
r_{21} & r_{22} & r_{23} \\
r_{31} & r_{32} & r_{33}
\end{array}\right)\left(\begin{array}{l}
x_{1} \\
x_{2} \\
x_{3}
\end{array}\right)+\left(\begin{array}{l}
t_{1} \\
t_{2} \\
t_{3}
\end{array}\right)
$$

soit $\vec{x}^{\prime}=\mathrm{R} \vec{x}+\vec{t}$.

L'opération est désignée par le symbole abrégé $(\mathrm{R}, \vec{t})$ connu sous le nom de notation de Seitz.

Rotations

La matrice $\mathrm{R}$ est à une opération d'ordre $\mathrm{n}$ fini, c'est-à-dire que son application successive $\mathrm{n}$ fois correspond à l'identité. Elle peut prendre 3 formes différentes, illustrées dans la figure ci-dessous :

$$
\text { rotation } A_{n}\left(\begin{array}{ccc}
\cos \phi-\sin \phi & 0 \\
\sin \phi & \cos \phi & 0 \\
0 & 0 & +1
\end{array}\right) \text { rotoréflexion } S_{n}\left(\begin{array}{ccc}
\cos \phi-\sin \phi & 0 \\
\sin \phi & \cos \phi & 0 \\
0 & 0 & -1
\end{array}\right) \text {, roto-inversion } \mathrm{I}_{\mathrm{n}}\left(\begin{array}{ccc}
-\cos \phi & \sin \phi & 0 \\
-\sin \phi-\cos \phi & 0 \\
0 & 0 & -1
\end{array}\right)
$$

avec $\phi=2 \pi / \mathrm{n}$.
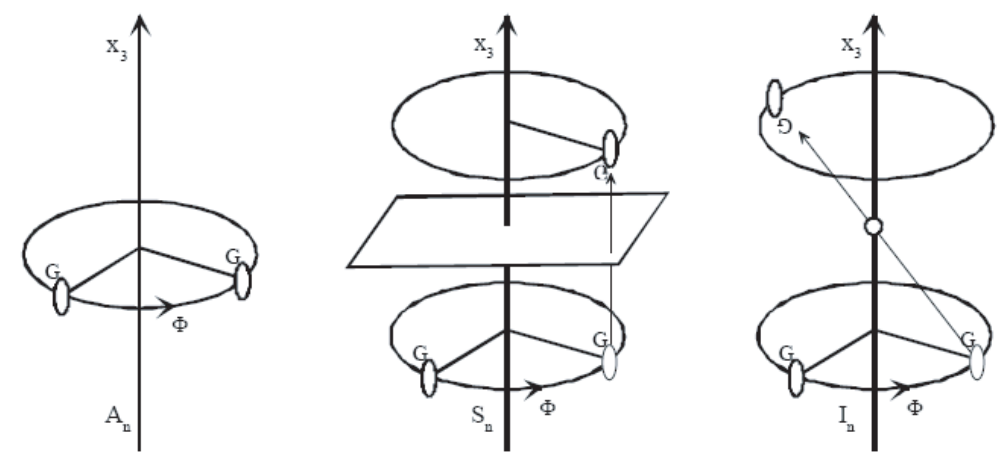

Figure 3. Représentation schématique des trois opérations : rotation, roto-réflexion, roto-inversion. 
La rotation $\left(A_{n}\right)$ d'ordre $n$ est une opération de première espèce, qui conserve la chiralité. La roto-réflexion $S_{n}$ peut être vue comme la combinaison d'une rotation et d'une symétrie suivant un plan perpendiculaire à l'axe de rotation. La roto-inversion $I_{n}$ peuvent être vue comme une rotation suivie d'une symétrie (inversion) par rapport à un point sur l'axe de rotation. $\mathrm{S}_{\mathrm{n}}$ et $\mathrm{I}_{\mathrm{n}}$ sont des opérations de deuxième espèces, qui ne conservent pas la chiralité (elles transforment une main droite en une main gauche). On voit facilement que ces deux descriptions sont équivalentes car :

$$
\mathrm{I}(\phi)=\mathrm{S}(\pi+\phi) .
$$

On peut donc décrire les opérations de deuxième espèce selon l'une ou l'autre. Le système de Schoenflies est basé sur les roto-réflexions. Le système adopté dans les tables internationales de Cristallographie est celui de Hermann-Mauguin, basé sur les roto-réflexions.

Dans les cristaux, il n'est pas possible d'avoir des rotations/roto-réflexions d'ordre n quelconques. Seules les valeurs $n=1,2,3,4$ et 6 . Pour les rotations, $A_{1}$ correspond à l'identité $E, A_{2}, A_{3}, A_{4}$ et $A_{6}$ à des rotations autour d'un axe de $2 \pi / \mathrm{n}$, notées axes $2,3,4,6 . \mathrm{I}_{1}$ correspond à l'application d'un centre d'inversion ( $\overline{1}), \mathrm{I}_{2}$ à un miroir $(\mathrm{m})$, et $\mathrm{I}_{3}, \mathrm{I}_{4}, \mathrm{I}_{6}$ à des rotation autour d'un axe de $2 \pi / \mathrm{n}$, suivie d'une inversion (axes $\overline{3}, \overline{4}, \overline{6}$, cf figure ci-dessous).
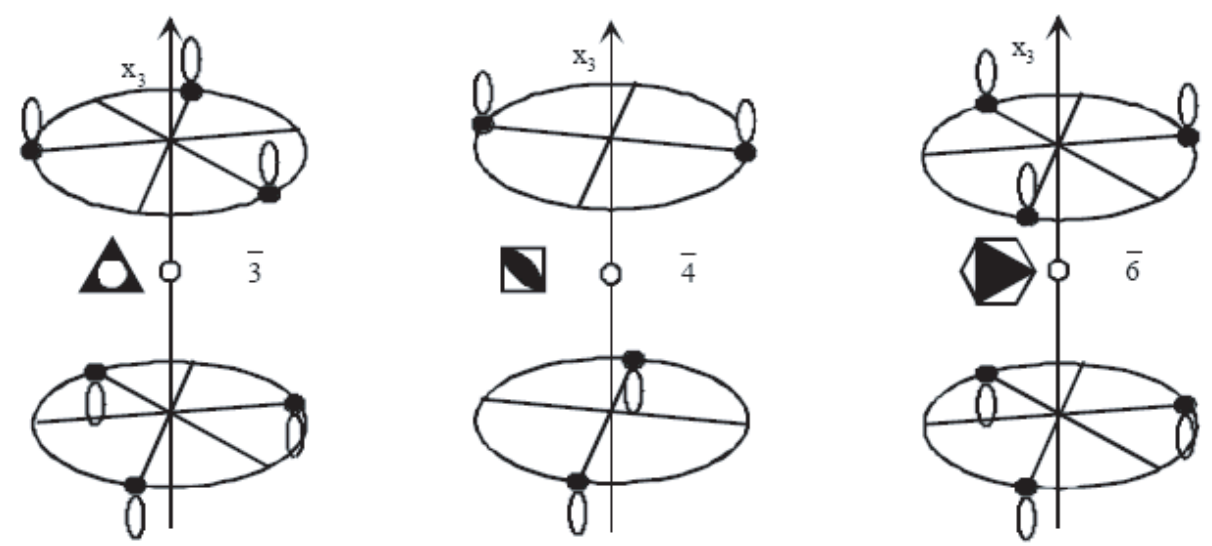

Figure 4. Représentation schématique des axes de roto-inversion $\overline{3}, \overline{4}, \overline{6}$.

\section{Translations}

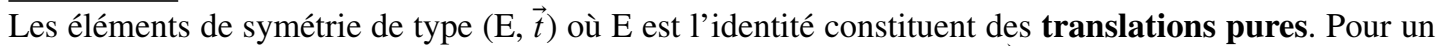
cristal, $\vec{t}$ est nécessairement un vecteur du réseau et s'écrit : $\vec{t}=u \vec{a}+v \vec{b}+w \vec{c}$.

Rotation-translations:

Les éléments de symétrie associant une rotation ou une roto-réflexion et une translation sont les axes hélicoïdaux et les miroirs à glissement. La translation est toujours effectuée dans une direction parallèle à l'axe de rotation (cf. figure 5). Du fait de la translation, ces éléments de symétrie ne laissent pas de point invariant.

\subsection{Groupes de symétrie dans les cristaux}

Un groupe mathématique $G$ est l'association d'un ensemble d'objets et d'une opération (notée $x$ ) sur ces objets, répondant aux critères suivant :

- existence d'un élément neutre $e$ dans $G$ tel que $\forall a \in G$, axe $=$ exa $=a$

- associativité $(\forall a, b, c \in G a x b x c=(a x b) x c=a x(b x c))$

- tout élément $a$ possède un inverse noté $a^{-1}$ tel que $a x a^{-1}=a^{-1} x a=e$. 


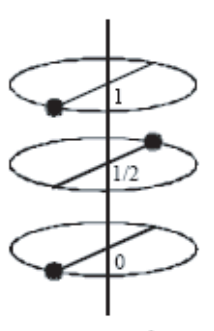

$2_{1}$

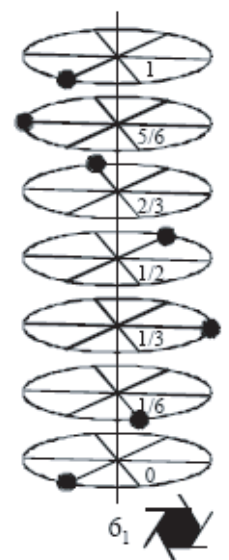

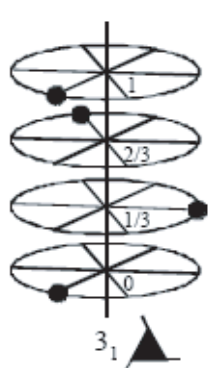

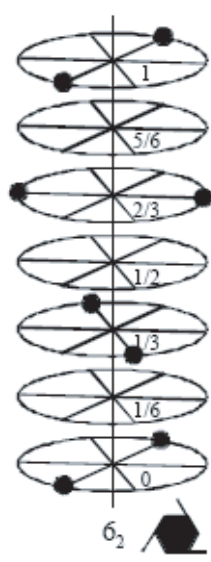

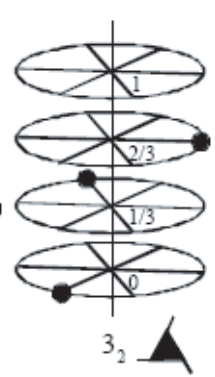

$3_{2}$ A

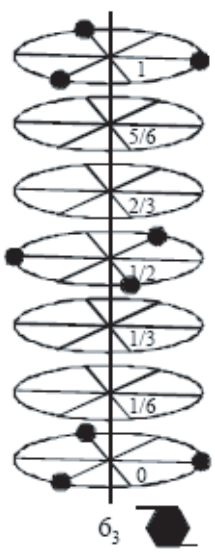

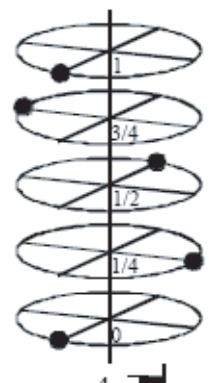

$4_{1}$ 巫
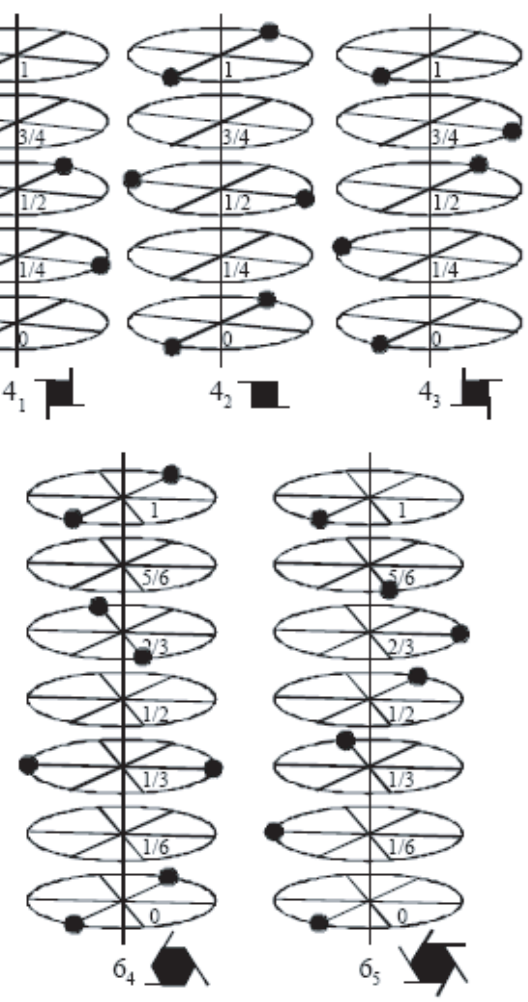

Figure 5. Représentation schématique des axes hélicoïdaux.

Pour les éléments de symétrie définis ci-dessus, on peut construire des groupes pour l'opération «combinaison de symétries » qui consiste à appliquer successivement les opérations de symétries de deux de ses éléments. Pour deux éléments $\left(\mathrm{Q}, \vec{t}_{Q}\right)$ et $\left(\mathrm{P}, \vec{t}_{P}\right)$, on a :

$$
\begin{array}{r}
\vec{x}^{\prime \prime}=Q \vec{x}^{\prime}+\vec{t}_{Q}=Q\left(P \vec{x}+\vec{t}_{P}\right)+\vec{t}_{Q}=Q P \vec{x}+Q \vec{t}_{P}+\vec{t}_{Q} \text { soit : } \\
\left(Q, \vec{t}_{Q}\right) x\left(P, \vec{t}_{P}\right)=\left(Q P, Q \vec{t}_{P}+\vec{t}_{Q}\right) .
\end{array}
$$

Notons que cette opération n'est en général pas commutative.

À partir de la combinaison des opérations de symétries cristallographiques définies ci-dessus et de cette opération, on montre qu'on peut construire $\mathbf{2 3 0}$ groupes de symétrie différents appelés groupes d'espace, dont la description est donnée dans les Tables Internationales de Cristallographie. Chaque groupe d'espace est un groupe clos d'ordre fini, qui est complètement défini par la connaissance d'un petit nombre de ses éléments, appelés éléments générateurs. Chaque élément du groupe peut être obtenu par combinaison finie des éléments générateurs du groupe. La connaissance du groupe d'espace définit complètement la symétrie du cristal et de l'ensemble de ses propriétés physiques et structurales.

Du fait de la symétrie du groupe d'espaces, de nombreux objets équivalents par symétrie vont se trouver à l'intérieur d'une même maille. On appelle unité asymétrique la plus petite partie de la maille (ou groupe d'atomes, motif) qui permet de générer l'ensemble du cristal par la seule application des opérations de symétrie.

Les groupes constitués uniquement d'éléments de symétrie sans translation (de type $(\mathrm{R}, \overrightarrow{0})$ ) sont appelés groupes ponctuels, car ils laissent au moins un point de l'espace invariant. Il en existe 32, qui constituent un sous ensemble des 230 groupes d'espace. Les symétries des groupes ponctuels sont celles des propriétés macroscopiques des cristaux, notamment de leurs formes externes. 


\begin{tabular}{|c|c|c|c|}
\hline Systèmes cristallins & Groupe ponct & tuels & Groupes d'espace \\
\hline Triclinique & $\begin{array}{l}1 \\
1 \\
\end{array}$ & & $\begin{array}{l}\mathrm{P} 1 \\
\mathrm{P} \overline{1}\end{array}$ \\
\hline Monoclinique & $\begin{array}{c}2 \\
\mathrm{~m} \\
2 / \mathrm{m}\end{array}$ & $\square$ & $\begin{array}{l}\mathrm{P} 2 \mathrm{P}_{2}{ }_{1} \mathrm{C} 2 \\
\mathrm{Pm} \mathrm{Pc} \mathrm{Cm} \mathrm{Cc} \\
\mathrm{P} 2 / \mathrm{m} \mathrm{P}_{2} / \mathrm{m} \mathrm{C} 2 / \mathrm{m} \mathrm{P}_{2} / \mathrm{CP}_{1} / \mathrm{c} \mathrm{C} 2 / \mathrm{c}\end{array}$ \\
\hline Orthorhombique & $\begin{array}{c}222 \\
\mathrm{~mm} 2\end{array}$ & & 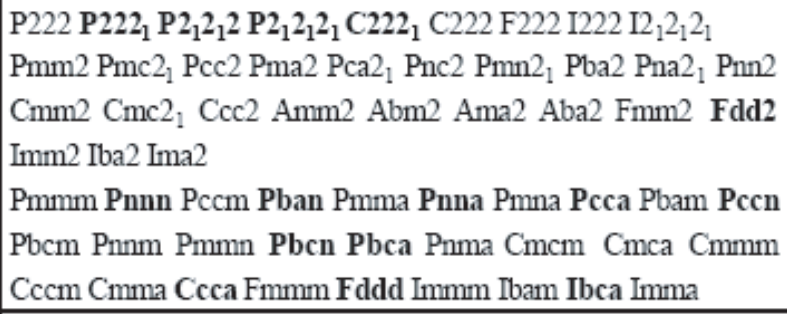 \\
\hline Tétragonal & $\begin{array}{c}4 \\
\overline{4} \\
4 / \mathrm{m} \\
422 \\
4 \mathrm{~mm} \\
\overline{4} \mathrm{~m} \\
4 / \mathrm{mmm}\end{array}$ & & 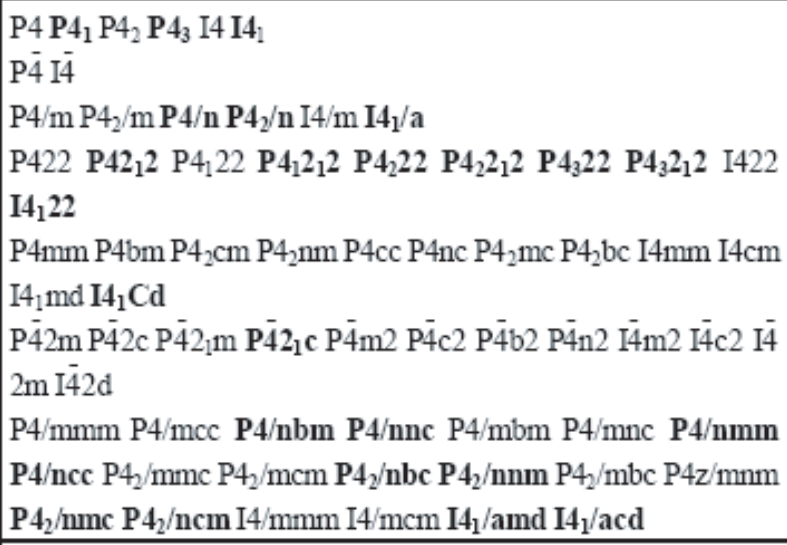 \\
\hline Rhomboédrique & $\begin{array}{c}3 \\
\overline{3} \\
32 \\
3 \mathrm{~m} \\
\overline{3} \mathrm{~m}\end{array}$ & & 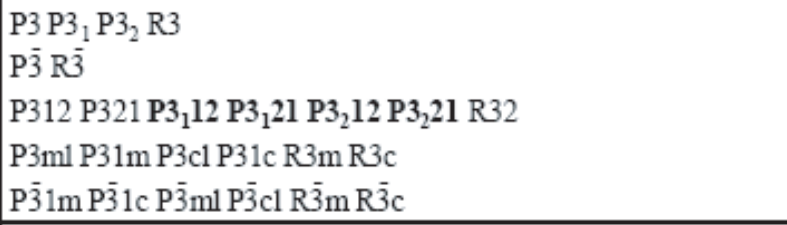 \\
\hline Hexagonal & $\begin{array}{c}6 \\
\overline{6} \\
6 / \mathrm{m} \\
622 \\
6 \mathrm{~mm} \\
\overline{6} \mathrm{~m} \\
6 / \mathrm{mmm} \\
\end{array}$ & & 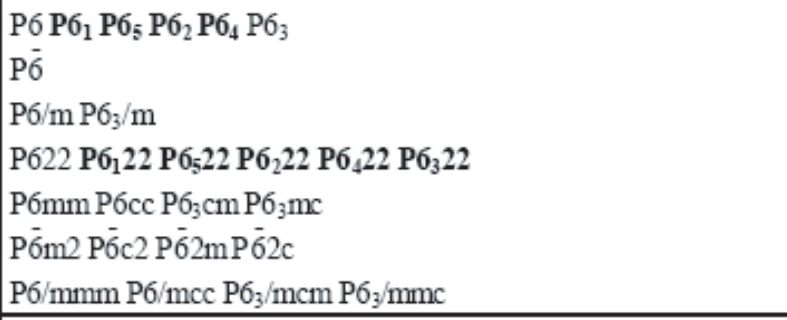 \\
\hline Cubique & $\begin{array}{l}23 \\
\mathrm{~m} \overline{3} \\
432 \\
\overline{4} 3 \mathrm{~m} \\
\mathrm{~m} \overline{3} \mathrm{~m}\end{array}$ & & 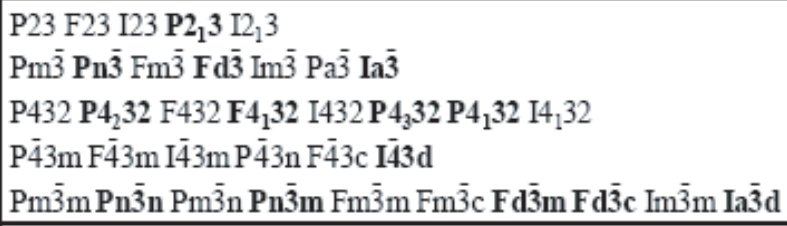 \\
\hline
\end{tabular}

Figure 6. Les 7 systèmes cristallins, 32 groupes ponctuels et 230 groupes d'espace. 
Le centre d'inversion joue parmi l'ensemble des symétries un rôle particulier : toutes les propriétés physiques d'un cristal centrosymétrique (et notamment les phénomènes de diffraction qu'il produit) sont représentées par des fonctions paires. On regroupe donc tous les groupes ponctuels qui ne se distinguent que par l'existence d'un centre d'inversion dans une même classe de Laüe. Il en existe 11, notées selon le symbole du groupe ponctuel centrosymétrique qu'elles représentent.

\section{DIFFRACTION, RÉSEAU RÉCIPROQUE}

\subsection{Introduction}

Les atomes, objet dont la taille est de l'ordre de l'angstroem, vont diffuser les rayonnements dont la longueur d'onde est voisine. L'interaction responsable de la diffusion va dépendre du type de rayonnement : électromagnétique pour les rayons $\mathrm{X}$, nucléaire pour les neutrons (cf. le cours de G. Heger). En cristallographie, on s'intéresse essentiellement à la composante cohérente et élastique du rayonnement diffusé. Un cristal étant un objet périodique constitué d'atomes, celle-ci va en effet donner lieu à un phénomène d'interférences, appelé diffraction du rayonnement par le cristal et caractérisé par l'existence de directions très précises dans lesquelles l'intensité diffractée est localisée. La mesure des angles de diffraction va renseigner sur les caractéristiques métriques du cristal (système cristallin, maille du réseau), la mesure de leurs intensités permettra de remonter à la structure du motif cristallin (de l'unité asymétrique).

Un cristal peut être décrit comme la convolution d'un motif d'atome $M(\vec{r})$ et d'un réseau $R(\vec{r})$ :

$$
C(\vec{r})=R(\vec{r}) * M(\vec{r}) .
$$

L'amplitude diffractée correspond à la transformée de Fourier de l'objet diffusant. En utilisant le théorème de convolution, il vient :

$$
C(\vec{h})=\mathrm{TF}\{C(\vec{r})\}=\operatorname{TF}\{R(\vec{r}) * M(\vec{r})\}=R(\vec{h}) \times M(\vec{h}) .
$$

Le terme $R(\vec{h})$ représente la transformée de Fourier du réseau. C'est également un réseau tridimensionnel appelé réseau réciproque. Le terme $M(\vec{h})$ est appelé facteur de structure. Il représente la transformée de Fourier de la structure atomique d'une maille du cristal. La connaissance du réseau réciproque et des facteurs de structure détermine complètement l'arrangement atomique au sein du cristal.

\subsection{Réseau réciproque}

Le réseau réciproque d'un cristal est obtenu par transformée de Fourier de son réseau direct. Les vecteurs de base du réseau réciproque $\left(\vec{a}^{*}, \vec{b}^{*}, \vec{c}^{*}\right)$ sont reliés à ceux du réseau direct $(\vec{a}, \vec{b}, \vec{c})$ par :

$$
\vec{a}^{*}=\frac{\vec{b} \wedge \vec{c}}{V}, \quad \vec{b}^{*}=\frac{\vec{c} \wedge \vec{a}}{V}, \quad c^{*}=\frac{\vec{a} \wedge \vec{b}}{V} \quad \text { où } \quad V=\vec{a} \wedge \vec{b} \cdot \vec{c} \text { est le volume de la maille. }
$$

Un vecteur du réseau réciproque s'écrit: $\vec{h}=h \vec{a}^{*}+k \vec{b}^{*}+l \vec{c}^{*}$, avec $(h, k, l)$ entiers appelés indices de Miller du nœud pointé par le vecteur $\vec{h}$.

Lors d'une expérience de diffraction avec un faisceau incident monochromatique de vecteur d'onde $\vec{k}_{0}$, la diffraction a lieu dans les directions des vecteurs de diffusion $\vec{k}$ tels que $\vec{k}=\vec{k}_{0}+\vec{h}$. Les deux vecteurs d'onde ayant pour module $1 / \lambda$, il en résulte une condition reliant le module de $\vec{h}$, noté $d^{*}$, à l'angle de diffraction entre $\vec{k}_{0}$ et $\vec{k}$, noté $2 \theta$ qui s'écrit :

$$
\sin (\theta)=\frac{d^{*} \cdot \lambda}{2} \Leftrightarrow 2 d \cdot \sin (\theta)=\lambda \text { où } d=1 / d^{*}: \text { c'est la loi de Bragg. }
$$

Cette condition est illustrée dans la construction d'Ewald représentée ci-dessous. 


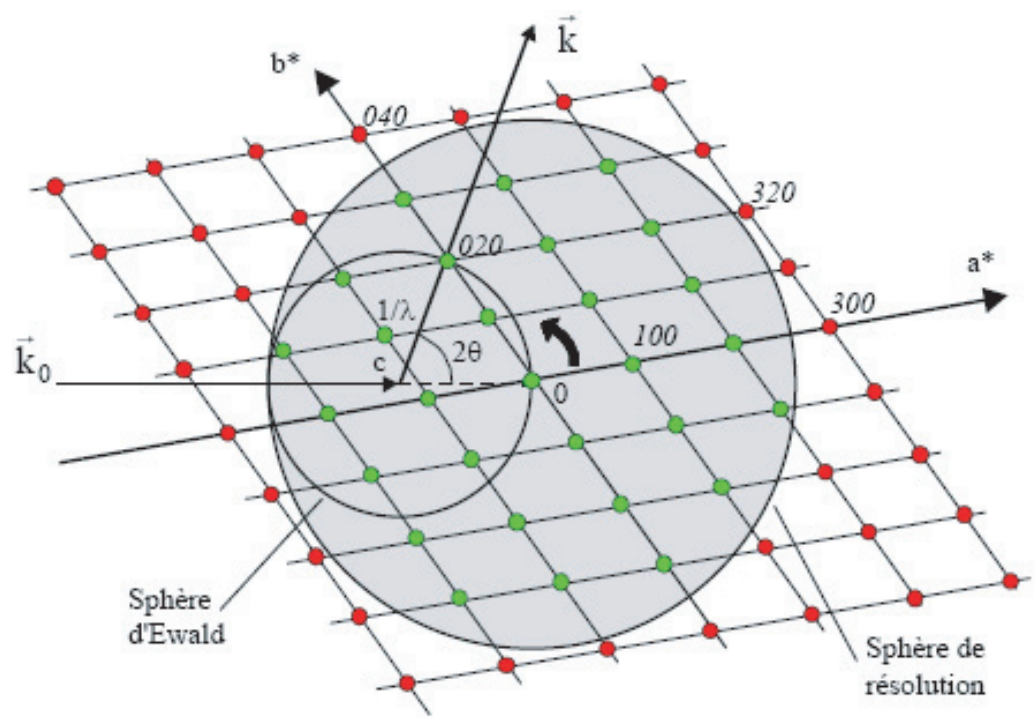

Figure 7. Construction d'Ewald.

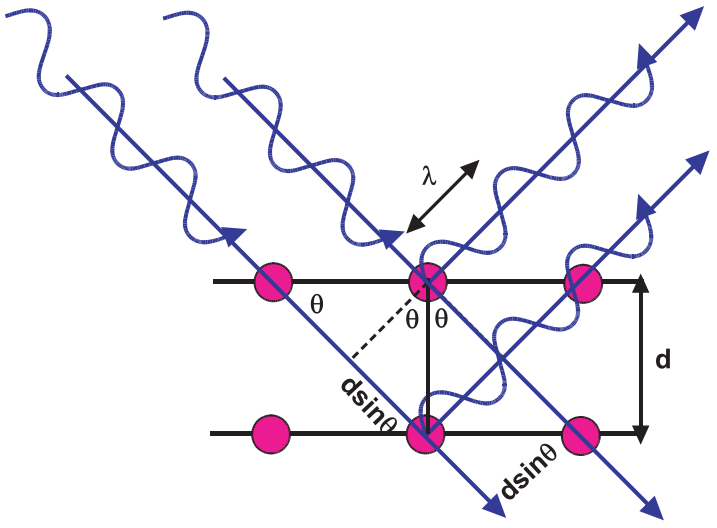

Figure 8. Illustration de la condition de Bragg.

La diffraction a lieu lorsque un nœud du réseau (ici (020)) intersecte la sphère d'Ewald. Celle-ci a pour rayon $1 / \lambda$ et pour centre l'intersection des vecteurs d'onde incident et diffracté. Le réseau réciproque tourne autour de son origine fixée à l'intersection de la direction du vecteur d'onde incident et de la sphère. Seuls les nœuds situés à l'intérieur de la sphère de résolution de rayon $2 / \lambda$ pourront donner lieu à la diffraction.

Tout vecteur $\vec{h}$ d'indices $(h k l)$ appartenant au réseau réciproque est perpendiculaire à une famille de plans du réseau direct parallèles entre eux, d'équation $h x+k y+l z=0$ et notée [hkl]. La distance inter-réticulaire $\boldsymbol{d}$ intervenant dans la loi de Bragg ci-dessus n'est autre que la distance entre 2 plans consécutifs de la famille. Elle est reliée aux paramètres de maille du réseau direct et aux indices $(h k l)$ de la famille de plans. Ainsi, la loi de Bragg peut être réinterprétée comme la condition d'interférence pour la famille de plans $[\mathrm{hkl}]$ de distance interréticulaire $d$, comme représenté sur la figure 8 .

Du fait de la forme et des dimensions finies du cristal, dans la pratique il existe un petit volume autour des nœuds du réseau réciproque pour lequel on observe le phénomène de diffraction (figure 9). Ces volumes sont obtenus par transformée de Fourier du domaine (forme) limitant le cristal. 


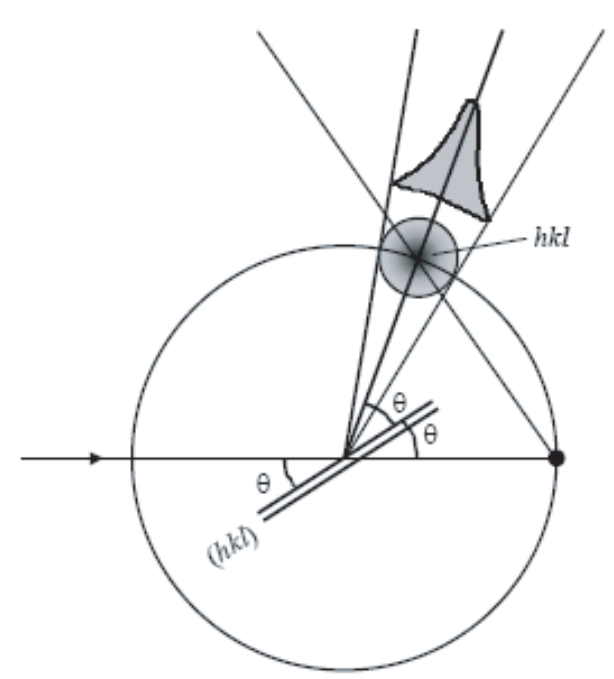

Position de réflexion sélective $h k l$

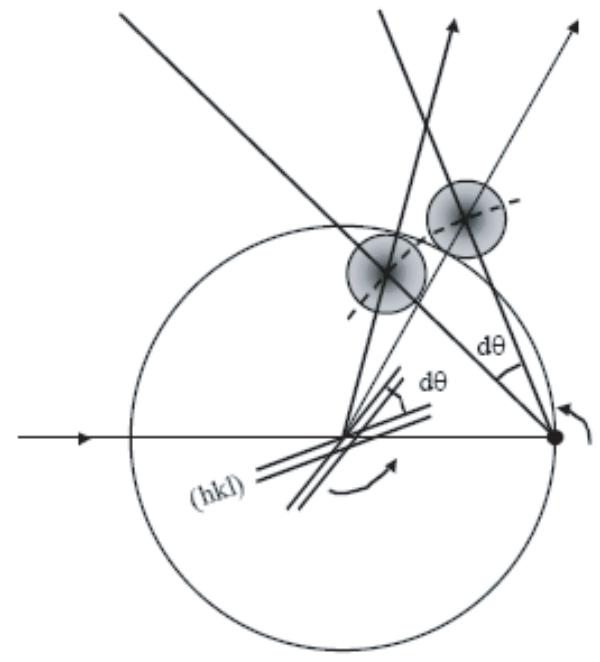

Positions extrêmes conduisant à une intensité non nulle

Figure 9. Construction d'Ewald montrant la relaxation de la loi de Bragg du au domaine diffractant autour de chaque nœud du réseau réciproque.

\subsection{Facteur de structure, intensité diffractée}

Dans le calcul de l'amplitude diffractée par un cristal, le terme $M(\vec{h})$, transformée de Fourier du contenu atomique de la maille est le facteur de structure $\boldsymbol{F}_{\boldsymbol{h} k l}$. Il s'écrit :

$$
F_{h k l}=\sum_{j \subset c e l l} f_{j} \exp \left(2 i \pi\left(h x_{j}+k y_{j}+l z_{j}\right)\right)
$$

où : $f_{j}$ : facteur de diffusion de l'atome $\mathrm{j}$ de coordonnées $\left(x_{j}, y_{j}, z_{j}\right)$. Pour les rayons $\mathrm{X}, \boldsymbol{f}_{j}$ est le facteur de forme de l'atome, transformée de Fourier de sa densité électronique. Du fait de l'étendue du nuage électronique dans l'espace direct, le facteur de forme va décroître rapidement en fonction de l'angle de Bragg de la réflexion $(h k l)$. Pour les neutrons, où l'interaction a lieu avec le noyau quasi ponctuel, la grandeur correspondante est la longueur de fermi, qui reste constante en fonction de $\theta$.

En fait, un atome ne reste pas immobile sur sa position, mais subit un mouvement de vibration lié à la présence des phonons dans le cristal, et d'autant plus important que la température est élevée. L'expérience de diffraction réalise une moyenne spatiale et temporelle des positions atomiques dans l'ensemble du cristal, sur un temps très grand devant ces fréquences de vibration. Tout ce passe comme si on observait une densité de diffusion atomique plus faible que celle de l'atome fixe isolé. Pour prendre en compte cet effet on apporte une correction au facteur de diffusion atomique, en le multipliant par un terme de Debye-Waller dépendant d'un paramètre de déplacement atomique (a.d.p.). Le facteur de structure s'écrit maintenant :

$$
F_{h k l}=\sum_{j \subset c e l l} g_{j} \exp \left(2 i \pi\left(h x_{j}+k y_{j}+l z_{j}\right)\right)
$$

avec $g_{j}=f_{j} \cdot T_{j}$ et $T_{j}=\exp \left\{-B_{j}\left(\sin ^{2} \theta\right) / \lambda^{2}\right\}$ terme de Debye-Waller dans le cas de vibrations harmoniques isotropes. $B_{j}=8 \pi^{2}\left\langle U_{j}^{2}\right\rangle$ et $\left\langle U_{j}^{2}\right\rangle$ est le déplacement quadratique moyen de l'atome $\mathrm{j}$. 


\begin{tabular}{|c|c|c|c|c|c|c|c|}
\hline $\begin{array}{l}\text { Réfle- } \\
\text { xions }\end{array}$ & $\begin{array}{l}\text { Conditions } \\
\text { d'observation }\end{array}$ & \begin{tabular}{|l} 
Elément de symétrie; \\
orientation
\end{tabular} & Translations & $\begin{array}{l}\text { Réfle- } \\
\text { xions }\end{array}$ & $\begin{array}{l}\text { Conditions } \\
\text { d'observation }\end{array}$ & $\begin{array}{l}\text { Type de réseau, ou } \\
\text { élément de symétrie }\end{array}$ & Translations \\
\hline \multirow[t]{2}{*}{$h 00$} & $h=2 n$ & hélices $2,4_{2} ;[100]$ & $\frac{1}{2} \mathbf{a}$ & $h k l$ & $h+k+l=2 n$ & réseau I & $\frac{1}{2}(a+b+c)$ \\
\hline & $h=4 n$ & hélices $4_{1}, 4_{3} ;[100]$ & $\frac{1}{4} \mathbf{a}$ & & $h+k=2 n$ & réseau C & $\frac{1}{2}(\mathbf{a}+\mathbf{b})$ \\
\hline \multirow[t]{3}{*}{$0 k 0$} & $k=2 n$ & hélices 21,$42 ;[010]$ & $\frac{1}{2} \mathbf{b}$ & & $h+l=2 n$ & réseau B & $\frac{1}{2}(\mathbf{a}+\mathbf{c})$ \\
\hline & $k=4 n$ & hélices $44_{1}, 43 ;[010]$ & $\frac{1}{4} \mathbf{b}$ & & $k+l=2 n$ & réseau A & $\frac{1}{2}(\mathbf{b}+\mathbf{c})$ \\
\hline & $l=2 n$ & hélices $2,4_{2}, 6_{3} ;[001]$ & $\frac{1}{2} \mathbf{c}$ & & $\left.\begin{array}{l}h, k, l \text { tous pairs } \\
\text { ou tous impairs }\end{array}\right\}$ & réseau $F$ & $\frac{1}{2}(\mathbf{a}+\mathbf{b}) \cdot \frac{1}{2}(\mathbf{a}+\mathbf{c}) \cdot \frac{1}{2}(\mathbf{b}+\mathbf{c})$ \\
\hline \multirow{4}{*}{$00 l$} & $l=3 n$ & {$\left[\begin{array}{l}\text { helices } z_{1}, 4_{2}, 6_{3} ;[001] \\
\text { hélices } 3,3_{2}, 6_{2}, 6_{4}:[001]\end{array}\right.$} & $\frac{1}{2} \mathbf{c}$ & & $-h+k+l=3 n$ & réseau R (reverse) & $\frac{1}{3}(2 \mathbf{a}+\mathbf{b}+\mathbf{c}) \cdot \frac{1}{3}(\mathbf{a}+2 \mathbf{b}+2 \mathbf{c})$ \\
\hline & $l=3 n$ & hélices $3_{1}, 3_{2}, 6_{2}, 6_{4}$; [001] & $\frac{1}{3} \mathrm{c}$ & & $h-k+l=3 n$ & réseau R (obverse) & $\frac{1}{3}(\mathbf{a}+2 \mathbf{b}+\mathbf{c}) \cdot \frac{1}{3}(2 \mathbf{a}+\mathbf{b}+2 \mathbf{c})$ \\
\hline & $l=4 n$ & hélices 4 , 4 4; [001] & $\frac{1}{4} \mathbf{c}$ & $0 k l$ & $k=2 n$ & plan b, (100) & $\frac{1}{2} \mathbf{b}$ \\
\hline & $l=6 n$ & hélices 6 ,, $6_{5} ;[001]$ & $\frac{1}{6} \mathbf{c}$ & & $l=2 n$ & plan $c,(100)$ & $\frac{1}{2} \mathbf{c}$ \\
\hline \multirow[t]{13}{*}{$h h 0$} & $h=2 n$ & hélice $2_{1} ;[110]$ & $\frac{1}{2}(\mathbf{a}+\mathbf{b})$ & & $k+l=2 n$ & plan $n,(100)$ & $\frac{1}{2}(\mathbf{b}+\mathbf{c})$ \\
\hline & & & & & $k+l=4 n$ & plan d, (100) & $\frac{1}{4}(b+c)$ \\
\hline & & & & $h 0 l$ & $h=2 n$ & plan a, (010) & $\frac{1}{2} \mathbf{a}$ \\
\hline & & & & & $l=2 n$ & plan c, (010) & $\frac{1}{2} \mathrm{c}$ \\
\hline & & & & & $h+l=2 n$ & plan n, (010) & $\frac{1}{2}(a+c)$ \\
\hline & & & & & $h+l=4 n$ & plan d, (010) & $\frac{1}{4}(a+c)$ \\
\hline & & & & $h k 0$ & $h=2 n$ & plan a, (001) & $\frac{1}{2} \mathbf{a}$ \\
\hline & & & & & $k=2 n$ & plan b, (001) & $\frac{1}{2} \mathbf{b}$ \\
\hline & & & & & $h+k=2 n$ & plan n, (001) & $\frac{1}{2}(\mathbf{a}+\mathbf{b})$ \\
\hline & & & & & $h+k=4 n$ & plan d, (001) & $\frac{1}{4}(\mathbf{a}+\mathbf{b})$ \\
\hline & & & & hhl & $l=2 n$ & plan c, (1T0) & $\frac{1}{2} \mathrm{c}$ \\
\hline & & & & & $2 h+l=2 n$ & plan n, (1T0) & $\frac{1}{2}(\mathbf{a}+\mathbf{b}+\mathbf{c})$ \\
\hline & & & & & $2 h+l=4 n$ & plan d, (1T0) & $\frac{1}{4}(a+b+c)$ \\
\hline
\end{tabular}

Figure 10. Conditions de présence dues aux éléments de symétrie avec translation.

Pour chaque réflexion $(h k l)$, la grandeur accessible à la mesure est en fait l'intensité diffractée. Dans l'approximation cinématique où on néglige la diffraction multiple, elle s'écrit :

$$
I_{h k l} \cdot=S \cdot C_{h k l} \cdot\left|\mathrm{F}_{\mathrm{hkl}}\right|^{2}
$$

où $\mathrm{S}$ est un facteur d'échelle et $C_{h k l}$ un terme de corrections expérimentales. Ces termes comprennent à la fois des corrections dues à la géométrie de l'instrument et aux caractéristiques du rayonnement (terme de Lorentz-polarisation, multiplicité) et des effets liés à l'interaction rayonnement-matière dans l'échantillon (absorption, extinction, microstructure....).

\section{3. ÉTUDIER LA STRUCTURE CRISTALLOGRAPHIQUE AVEC LA DIFFRACTION}

\subsection{Introduction}

Les techniques de diffraction sont les méthodes les plus efficaces et les plus utilisées en cristallographie. Pour déterminer une structure cristalline, il faut déterminer les différentes composantes de celle-ci : le réseau (système cristallin, réseau de Bravais, paramètres de maille), l'ensemble des éléments de symétrie du cristal (i.e. son groupe d'espace) et le contenu de la maille, positions et facteurs de déplacement des atomes (i.e. les facteurs de structures). Tout un ensemble de techniques d'analyse et d'instruments basés sur la diffraction ont été développés pour obtenir ces informations. Elles se divisent en deux grandes familles suivant le type d'échantillon : monocristal ou poudre. Dans le cas d'un monocristal, on pourra accéder à une mesure tridimensionnelle du réseau réciproque, alors que pour une poudre, du fait de la moyenne réalisée par la répartition statistiquement aléatoire des orientations des grains, on observe qu'une projection sur une dimension de celui-ci. 


\subsection{Maille élémentaire, système cristallin}

La détermination de la maille élémentaire est obtenue à partir de la position des réflexions de Bragg. Dans le cas d'un monocristal, on a directement accès à la mesure des vecteurs du réseau réciproque et donc très facilement à la maille et au système cristallin. Pour les poudres, on ne dispose que des angles de Bragg des réflexions, et on doit donc résoudre un système d'équations non linéaires du type

$$
d_{h k l}=f(h, k, l, a, b, c, \alpha, \beta, \gamma)
$$

où $h, k, l$ sont les indices de Miller et $a, b, c, \alpha, \beta, \gamma$, les paramètres de maille à déterminer. Ce système n'a pas de solution unique et la résolution de ce problème est encore très difficile dans le cas de composés de basse symétrie et de grandes mailles, pour lesquels la superposition des réflexions de Bragg rend la mesure de leur position imprécise. Les différents logiciels d'indexation automatiques, travaillant dans l'espace direct ou réciproque, nécessitent des données de haute résolution soigneusement corrigées de tous les effets pouvant influer la position des réflexions (asymétrie, décalage de zéro...). Cela dit, une fois la maille obtenue et les réflexions du diagramme indéxées, la détermination de l'angle de Bragg est souvent plus précise pour une mesure sur poudre que sur monocristal, pour lequel la forme de la tache de diffraction peut être complexe. On peut donc obtenir plus aisément des paramètres de maille précis à partir de diffractogrammes de poudres que de données de monocristaux.

\subsection{Groupe d'espace}

La détermination du groupe d'espace est réalisée en observant les extinctions systématiques de classes de réflexions, liées à la présence d'éléments de symétrie avec translation (centrage du réseau, miroirs à glissements, axes hélicoïdaux). Par exemple, pour un réseau corps centré I, le vecteur $(\vec{a}+\vec{b}+\vec{c}) / 2$ est une translation du réseau. Le facteur de structure peut donc s'écrire :

$$
\begin{aligned}
F_{h k l} & =\sum_{j \subset c e l l} f_{j} \exp \left(2 i \pi\left(h x_{j}+k y_{j}+l z_{j}\right)\right) \\
F_{h k l} & =\sum_{j \subset 1 / 2 \text { cell }} f_{j} \exp \left(2 i \pi\left(h x_{j}+k y_{j}+l z_{j}\right)\right)\{1+\exp (i \pi(h+k+l))\}
\end{aligned}
$$

pour $h+k+l$ pair, le second terme vaut 2, sinon il est nul. Les réflexions (hkl) dont la somme des indices est impaire sont donc systématiquement nulles. Suivant qu'on a une translation de réseau, due à un miroir ou à un axe, les conditions d'extinctions dites respectivement intégrales, zonales ou sériales porteront sur 3, 2 ou 1 indices. Elles sont listées ci-dessous.

L'observation des extinctions systématiques permet en général de limiter le nombre de groupes d'espace possible. Seul un nombre relativement faible de groupes d'espaces peuvent être déterminés de façon univoque à partir des extinctions. Ils sont notés en gars dans la figure 6. Sinon, il faudra tenter de résoudre la structure dans chacun des groupes d'espaces compatibles avec les extinctions systématiques observées. Là encore, la détermination des conditions d'extinction est beaucoup plus aisée sur monocristaux que sur poudres, à cause des chevauchements de réflexions inhérents aux diffractogrammes de poudres.

\subsection{Structure du motif}

La difficulté fondamentale dans la détermination d'une structure par diffraction est le fait que les données cristallographiques disponibles sont liées seulement aux intensités des pics de Bragg, proportionnelles aux carrés des facteurs de structure. Ces derniers étant des nombres complexes $\left(F_{h k l}=\left|F_{h k l}\right| e^{i \alpha}\right.$, où $\alpha$ est la phase), l'information portée par la phase semble a priori être perdue. Afin de déterminer la structure à partir des données de diffraction, les phases des facteurs de structure 
doivent être redéterminées. Il existe diverses méthodes permettant d'obtenir les modèles conduisant aux phases et la liste suivante n'est pas exhaustive :

- Méthodes de Patterson

- Méthodes directes (Inégalités, F, U, et E, Invariants et Semi-invariants de structure, méthodes de probabilités...)

- Méthodes de l'atome lourd

- Méthodes de recherche (les méthodes par essais et erreurs, la recherche en rotation, la recherche en translation, les méthodes de superposition, de recuit simulé, d'algorythme génétique...).

Lorsque plusieurs atomes ont été localisés par l'une ou l'autre de ces méthodes, le modèle cristallographique formé est utilisé pour calculer les facteurs de structure $\left(\mathrm{F}_{c}\right)$, et ceux-ci serviront de sources de phases pour les calculs de synthèses de Fourier. Nous ne détaillerons ici que les deux techniques les plus communément utilisées, qui sont les méthodes de Patterson et les méthodes directes.

\subsubsection{La fonction de Patterson}

Si les facteurs de structure et leurs phases sont connus, la distribution de la densité électronique (ou de la densité liée aux noyaux dans le cas des neutrons) dans le volume de la maille élémentaire peut être calculée en utilisant l'expression générale :

$$
\rho(x, y, z)=\frac{1}{V} \sum_{h, k, l} F_{h k l} \exp (-2 i \pi(h x+k y+l z) .
$$

Cette série de Fourier correspond à la transformée de Fourier du facteur de structure $F_{h k l}$ mais des séries de Fourier cristallographiques peuvent être calculées avec diverses sortes de coefficients. L'une d'entre elles est la fonction de Patterson (ou fonction $|F|^{2}$ ) :

$$
P(u, v, w)=\frac{1}{V} \sum_{h} \sum_{k} \sum_{l}\left|F_{h k l}\right|^{2} \cos 2 \pi(h u+k v+l w) .
$$

Dans ce cas, les coefficients de la série de Fourier sont les carrés des facteurs de structure observés $\left|F_{o}\right|$. Comme ce sont des carrés de modules, ils sont sans phase et la fonction de Patterson peut être directement obtenue à partir des données de diffraction.

On peut montrer simplement que la fonction $P(u)$ correspond à l'autocorrélation de la densité électronique, soit :

$$
P(\mathbf{u})=\rho(\mathbf{r}) * \rho(-\mathbf{r})=\int \rho(\mathbf{r}) \rho(\mathbf{r}+\mathbf{u}) \mathrm{d} \mathbf{r}
$$

Tandis que la synthèse de Fourier usuelle, ayant les $F$ comme coefficients, représentait la distribution des atomes dans la maille, la carte calculée avec $|F|^{2}$ donne, elle, des pics correspondant à tous les vecteurs interatomiques. Ainsi, un pic situé au point $\mathbf{u}(u, v, w)$ sur une carte de Patterson signifie qu'il existe des atomes dans le cristal aux positions $\mathbf{r}_{1}\left(\mathrm{x}_{1}, \mathrm{y}_{1}, \mathrm{z}_{1}\right)$ et $\mathbf{r}_{2}\left(\mathrm{x}_{2}, \mathrm{y}_{2}, \mathrm{z}_{2}\right)$ telles que $\mathbf{u}=\mathbf{r}_{1}-\mathbf{r}_{2}$

$$
\begin{aligned}
u & =\mathrm{x}_{1}-\mathrm{x}_{2} \\
v & =\mathrm{y}_{1}-\mathrm{y}_{2} \\
w & =\mathrm{z}_{1}-\mathrm{z}_{2} .
\end{aligned}
$$

Le poids d'un pic de Patterson dépend des nombres d'électrons (rayons X) ou des facteurs de diffusion (neutrons) des atomes déterminant le vecteur, et on démontre qu'il est proportionnel au produit de leurs numéros atomiques ou de leurs facteurs de diffusion. C'est cette propriété qui permet de reconnaître sur une carte de Patterson, habituellement sans difficulté, les vecteurs liés à un atome lourd (à forte longueur de diffusion). On peut démontrer les propriétés de symétrie suivantes, pour la fonction de Patterson :

- Toutes les fonctions de Patterson sont centrosymétriques. 
- Leur type de réseau ( $P, I, C, F$, etc.) est le même que celui du groupe d'espace original de la structure.

- Leur groupe d'espace est dérivé du groupe d'espace original en remplaçant tous les éléments de symétrie avec translations (axes hélicoïdaux et plans de glissement) par les éléments correspondants sans translations (axes ordinaires et miroirs passant par l'origine), et en ajoutant un centre de symétrie s'il n'est pas déjà présent.

Malgré le problème du recouvrement des pics, des structures ont été résolues à partir de données de poudres au moyen des techniques de Patterson, et cette méthode semble moins sensible aux erreurs sur les intensités que les méthodes directes. Il n'est cependant pas forcément évident de reconstruire la liste des positions atomiques à partir de celle des vecteurs interatomiques, notamment à cause de la perte de l'origine de la structure dans le calcul de la fonction de Patterson.

\subsubsection{Les méthodes directes}

Les méthodes de calcul direct des phases sont maintenant largement employées et ont été automatisées à un point tel que la plupart des programmes fonctionnent en technique de "boite noire", dans laquelle les données brutes entrent d'un coté et la structure en partie résolue apparaît de l'autre. En fait, ceci n'est pas réellement vrai en diffraction des poudres qui une fois encore souffre du faible nombre de réflexions acceptables, à cause du recouvrement des pics.

Ces méthodes, qui atteignent aujourd'hui des complexités mathématiques très grandes, sont basées sur des hypothèses très simples :

- la densité électronique est positive partout dans l'espace

- les atomes sont des objets séparés, la densité électronique est fortement "piquée" à leurs positions,

- les amplitudes des facteurs de structure contiennent des l'information sur leurs phases.

Nous ne rentrerons pas ici dans le détail de ces méthodes forts complexes.

La relation de base des méthodes directes est l'équation de Sayre (1953), qui peut être simplement démontrée en appliquant le théorème de convolution à la transformée de Fourier du carré de la densité électronique. Elle s'écrit :

$$
E_{h k l}=T \sum_{h^{\prime}, k^{\prime}, l^{\prime}} E_{h^{\prime}, k^{\prime}, l^{\prime}} E_{h-h^{\prime}, k-k^{\prime}, l-l^{\prime}}
$$

où $E_{h k l}$ est le facteur de structure normalisé défini par : $E_{h k l}^{2} \approx \frac{\left|F_{h k l}\right|^{2}}{\sum_{c e l l} f_{j}^{2}}$.

Les triplets d'indices $(h 1, h 2, h 3)$ qui suivent la relation $h 1+h 2+h 3=0$ (appelée relation $\Sigma_{2}$ ) sont appelés triplets invariants de structure (c'est évidemment le cas pour $h 1=(h, k, l), h 2=\left(h^{\prime}, k^{\prime}, l^{\prime}\right)$ et $\left.h 3=\left(h-h^{\prime}, k-k^{\prime}, l-l^{\prime}\right)\right)$. Ils jouent un role clé dans les méthodes directes. En effet, les phases des facteurs de structures dépendent du choix de l'origine de la maille, ce qui n'est pas le cas de leurs amplitudes. Par conséquent, à partir des amplitudes, on ne peut obtenir d'information que sur des combinaisons de phases qui sont indépendantes du choix de l'origine : ce sont les invariants de structure.

Dans le cas centrosymétrique, les facteurs de structure sont des nombres réels, et le problème des phases se réduit à celui de la détermination du signe et simplifie grandement le problème.

$\mathrm{Si}\left|E_{h k l}\right|,\left|E_{h^{\prime}, k^{\prime}, l^{\prime}}\right|$ et $\left|E_{h-h^{\prime}, k-k^{\prime}, l-l^{\prime}}\right|$ sont assez grands, ils domineront la somme dans l'équation de Sayre, et il y aura une forte probabilité que $\mathrm{s}\left(E_{h k l}\right)=\mathrm{s}\left(E_{h^{\prime}, k^{\prime}, l^{\prime}}\right) \cdot \mathrm{s}\left(E_{h-h^{\prime}, k-k^{\prime}, l-l^{\prime}}\right)$, où $\mathrm{s}\left(E_{h k l}\right)$ représente le signe du facteur de structure. Si cette relation est vraie, la connaissance de deux des signes permet de déterminer le troisième. La probabilité que cette relation soit vraie peut être calculée par :

$$
p=1 / 2+(1 / 2) \tanh \left[\frac{1}{\sqrt{N}}|E(h 1) E(h 2) E(h 3)|\right]
$$

où $\mathrm{N}$ est le nombre d'atomes dans la maille. On voit que la probabilité augmente avec l'intensité des réflexions. Des relations similaires existent également dans le cas non centrosymétrique. 
Ces relations permettant d'obtenir (avec une certaine probabilité) la phase d'une réflexion à partir de celles de deux autres, il est nécessaire d'obtenir un jeu de phases de départ pour amorcer le mécanisme. Ce jeu (au moins 3 phases) est généralement déterminé par le choix de l'origine dans la maille élémentaire. Ce choix est, dans la plupart des cas, limité par la symétrie du groupe d'espace qui impose des restrictions quant à la position de l'origine dans la maille. C'est pourquoi la connaissance du groupe d'espace est absolument nécessaire avant l'application des méthodes directes pour la détermination de structure.

Pour chaque jeu de départ sélectionné, les phases des autres réflexions sont calculées en utilisant les relations entre phases d'invariants de structures. En fonction des divers choix possibles, un grand nombre de distributions de phases peut être obtenu. Comme on sait calculer les probabilités de véracité pour chaque phase, on peut caractériser chaque distribution de phases obtenue par une "figure de mérite". La figure de mérite la plus utilisée actuellement est la "figure de mérite combinée" CFOM, qui serait égale à 1 si toutes les phases étaient justes. Si CFOM $\ll 1$ pour tous les jeux de phases obtenus, la procédure est très vraisemblablement en échec. Si on obtient une distribution de phases pour laquelle $\mathrm{CFOM} \approx 1$, on peut obtenir une représentation de la structure obtenue en effectuant la transformée de Fourier des facteurs de structure normalisés, affectés des phases ainsi déterminées (E-MAP).

\subsubsection{Résolution dans l'espace direct}

Depuis quelques années sont apparues des techniques de résolution de structure travaillant dans l'espace direct. Ces méthodes sont particulièrement adaptées à la diffraction de poudre, où le problème est généralement mal conditionné, c'est-à-dire que le nombre d'observation est relativement faible devant le nombre de paramètres. Les données nécessaires pour utiliser ces techniques sont une liste d'intensités intégrées et une maille permettant de les indexer, ainsi que le contenu chimique de la maille. La connaissance du groupe d'espace n'est pas indispensable, bien qu'en général elle facilite beaucoup la solution et limite considérablement les temps de calcul.

Le principe général de ces méthodes est le suivant. On génère des modèles structuraux plausibles par déplacements des atomes (ou fragments de molécules) à partir d'un modèle initial quelconque, souvent tiré au hasard par un algorithme de type Monte Carlo. On compare le spectre calculé au spectre observé pour chaque modèle, et on ne garde que les plus plausibles. Les modèles suivants sont générés en modifiant certains des paramètres des modèles retenus précédemment. On itère le processus jusqu'à convergence (éventuelle !)

Il existe des façons diverses de modifier les paramètres dans le processus, qui conduisent à des méthodes variées telles que la recherche sur une grille (grid search), l'utilisation de l'algorithme génétique, le recuit simulé, le monte carlo inverse etc... A titre d'exemple nous allons décrire un peu plus en détail une méthode typique de recuit simulé, développée dans le programme Endeavour.

Exemple : Recuit simulé

À l'origine, il s'agit une méthode créée pour prédire les structures ab initio (sans données observées), à laquelle on a ajouté la prise en compte de données expérimentales de diffraction. Pour chaque configuration, on calcule une fonction de coût, basée sur une pseudo-énergie potentielle à minimiser, et sur l'accord expérience/calcul :

$$
C=\alpha E_{p o t}+(1-\alpha) R
$$

$E_{p o t}$ est un pseudo-potentiel dépendant du type de composé (ionique, covalent, intermétallique... ), qui peut être de type Lennard-Jones, ou coulombien, ou simplement basé sur la non interpénétration des atomes, à partir de distances interatomiques minimum acceptables. $\mathrm{R}$ est le résidu cristallographique décrivant l'accord entre le modèle et les données de diffraction :

$$
R=100 \sum_{2 \theta}\left|I_{\exp }(2 \theta)-I_{\text {calc }}(2 \theta)\right| / \sum_{2 \theta} I_{\exp }(2 \theta): \text { accord expérience/calcul. }
$$


On passe de la configuration $\mathrm{Ci}$ à $\mathrm{Ci}+1$ suivant un algorithme type Monte-Carlo, dans lequel les déplacements sont limités à une certaine amplitude. Au départ, cette amplitude est grande (typiquement de l'ordre de grandeur de la maille), puis elle est progressivement diminuée, ce qui est une façon de simuler un «refroidissement» d'un échantillon virtuel. Pour chaque déplacement, on recalcule la fonction de coût.

$\mathrm{Si}_{\mathrm{i}+1}<\mathrm{C}_{\mathrm{i}}$, déplacement accepté

$\mathrm{Si}_{\mathrm{i}+1}>\mathrm{C}_{\mathrm{i}}$, déplacement accepté avec la probabilité : $\exp \left[-\left(C_{i+1}-C_{i}\right) / K\right]$

$K$ est l'équivalent d'une pseudo-température, et décroît lentement au cours de la recherche suivant une courbe préétablie.

Les inconvénients de ces méthodes proviennent de la difficulté à définir de façon systématique un processus de calcul menant à la solution. Il est particulièrement difficile d'éviter les faux minimums lors de la convergence. C'est d'ailleurs une des raisons pour laquelle deux types de pseudo-énergie $E_{p o t}$ et $\mathrm{R}$ sont combinés dans Endeavour (les faux minimums des deux fonctions ont peu de chance a priori de coïncider, contrairement au vrai minimum !). Il est donc nécessaire d'introduire des connaissances non cristallographiques décrivant les potentiels d'interaction, qui sont en général mal connus. Il est également indispensable de connaître parfaitement le continu de la maille, contrairement aux méthodes directes, qui présentent une certaine tolérance dans ce domaine. De plus, ces techniques peuvent conduire à des temps de calcul considérables.

Cependant, elles peuvent s'avérer plus efficaces que les méthodes directes, notamment dans le cas de composés moléculaires, où l'ensemble de la molécule peut être décrit par un petit nombre de paramètres faisant intervenir les angles de torsions entre sous ensembles de la molécule, par exemple. Elles ont également l'avantage considérable de n'être pas limitées par la méconnaissance du groupe spatial, qui peut être redéterminé a posteriori si la structure a été résolue. De nombreux programmes existent à l'heure actuelle, dont la plupart sont malheureusement commerciaux (freeware : ESPOIR, Fullprof, Fox, commerciaux : Endeavour, PowderSolve, TOPAS, DASH ...). La plupart de ces programmes son accessibles à partir du site du CCP14 : http://www.ccp14.ac.uk/index.html.

\subsubsection{Affinement et cyclage de Fourier}

L'application des procédures de résolution décrite ci-dessus permet normalement d'obtenir un modèle grossier de la structure cristalline, correspondant en général aux positions des atomes de plus forts facteurs de diffusion de la structure, c'est-à-dire ceux qui contribuent le plus fortement aux intensités diffractées. Ensuite, des affinements par moindre carrés, à partir des intensités intégrées des réflexion de Bragg pour les monocristaux, où en prenant en compte le profil complet du diffractogramme pour les poudres (méthode de Rietveld) et des synthèses de Fourier sont effectués afin de localiser les atomes manquants.

Une autre fonction de Fourier importante est celle nommée "synthèse différence" ou encore "Fourier-différence". Dans ce cas, les coefficients sont définis comme :

$$
\Delta F=F_{o}-F_{c}
$$

où $F_{o}$ et $F_{c}$ sont les facteurs de structure observés et calculés. Le calcul de synthèses différence nécessite l'utilisation des phases des $F_{c}$ obtenues à partir du modèle structural affiné et appliquées à la fois à $F_{o}$ and $F_{c}$, les phases de $F_{o}$ n'étant pas connues. Les coefficients de Fourier sont alors définis comme :

$$
\Delta F=\left(\left|F_{o}\right|-\left|F_{C}\right|\right) e^{i \alpha} c
$$

$\left|F_{o}\right|$ et $\left|F_{C}\right|$ sont les modules des facteurs de structure observés et calculés, et $\alpha_{c}$ est la phase de $F_{c}$. L'expression de la fonction de synthèse différence est :

$$
\Delta \rho(x, y, z)=\frac{1}{V} \sum_{h} \sum_{k} \sum_{l} \Delta F e^{-2 \pi i(h x+k y+l z)} .
$$


Un avantage spécifique de cette synthèse différence provient du fait que, même lorsque les phases sont légèrement erronées, une carte de différence est capable de montrer les écarts entre le modèle structural proposé et la réalité, comme les atomes manquants par exemple. Ainsi, les nouveaux atomes révélés sur la carte différence sont introduits dans le modèle à affiner et la procédure est répétée jusqu'à ce que la structure soit complète. On pourra alors poursuivre l'affinement par moindre carrés des différents paramètres structuraux (positions atomiques, paramètres de déplacements) jusqu'à obtenir le meilleur accord entre le jeudes intensités observées et calculées. 